Neetles, porte-aiguilles, \&c. (I.iston's and Brodie's). 6. Varices. $a$. Including elastic bandages, stockings, anklets, \&c.

M. Autoplastic and Orthopedic Operations.-Special instruments for -1 . The various Taliacotian processes. a. Rhinoplasty. $\quad$. Chiloplasty, \&c. 2. Tenotomy and myotomy. a. Including long-bladed knives for the subcutaneous division of muscles and tendons, \&c. 3. Mechanical compensation of lost parts. a. Artificial eyes, noses, ears, chins, palates, teeth, hands, arms, legs, Sic. 4. Orthopedic processes. $\boldsymbol{a}$. Including all orthopedic apparatus, back and leg boards and irons, stays, belts, supports, suspenders, boots, shoes for the cure of bunions (Lanagan's), \&c.

N. Dressing Instruments. - a. Surgeon's pocket cases and their usual contents ; spring bistouries, tenaculums, scalpels, scissors, forceps, spatulie, probes and directors, needles, ligature-silks, \&c.

O. Miscellencous Philosophical Apparatus applicd to the Investigation and Treatment of Discase. - a . Microscopes, engiscopes and lenses, urinometers and thermometers; volta-electric apparatus, various illuminating speculums ; instruments in India rubber for the application of intense cold ; Hooper's water-cushions for the reception of fluids of any temperature; spongio-piline, patent lint, plasters, collodion, nipple-shields, breast-pumps, \&c.

P. Surgical Tables, Beds, Mattrasses, Chairs, Cradles, Rests, \&c.

Q. Post-mortem and Disscting Instruments, and Instruments for Einbalmment.

R. Instruments applicd to Veterinary Purposes.

\title{
INJURY TO THE SPINF.
}

[Communicated for the Boston Medical and Surgicn] Journal.]

Duning the severe storm which occurred about the middle of April, a large stable belonging to the Merrinac House, in Lawrence, was suddenly prostrated by the gale, burying a man and several horses in the ruins.

The man, Sewall Nute, 24 years of age, received a blow upon his back, and was thrown down so that his head and lower extremities came in contact, doubling the spinal column anteriorly upon itself, with a heavy weight resting upon him, severely compressing the internal organs and doing great violence to the spine. Shortly after the accident I was called, and, with Drs. Ames and Dana, made an examination in order to ascertain the nature and extent of the injury received by the patient. The skin had assumed a livid aspect, the pulse was slow and feeble, with rigors, and cold extremities. There was inability to support the head and chest, and the spine could not be elevated above an angle of $45 \mathrm{de}$ grees. On tracing the spine upward and counting the processes, we found the angle to be situated at the eighth dorsal vertebra, the superior surface of which could be distinctly felt ; the seventh, and those above it, being thrown forward, with a much larger space between the spinous processes of the seventh and eighth, than at any other point, and both were immovably fixed. The idea of a dislocation presented itself strongly 
to the mind, but as there was not complete paralysis, which usually occurs under such circumstances, and which is considered pathognomonic, we were cautious in regard to the diagnosis. Crepitus could not be detected, and consequently there was no certainty of a fracture, though there was a serious and perplexing deformity.

After a re-examination, we were unable to resist the conviction that there was a dislocation, and perhaps a fracture of such a character as to enlarge the spinal canal, and thus, by making the angle less acute, and the canal more capacious, preserve the spinal cord from severe compression.

In accordance with this view, two folded sheets were thrown around the patient and firmly secured, one above the hips and the other below the arms, and between them Jarvis's Surgical Adjuster was placed, and given in charge of an assistant. Gradual, but strong extending power was applied, until the spinal column was brought into a direct line and the deformity removed, the patient exclaiming that he was relieved. He was placed upon a rubber pillow, and carefully watched.

The night following, there was a high grade of febrile action, suppression of urine, and constipated bow'els, but these symptoms yielded to appropriate remedies. There was inflammation and extensive effusion in the injured part, which was removed by leeching and cold applications. He gradually improved, and on the 17th day was able to raise himself in bed. A spinal supporter was applied, and on the 24th day he was able to walk out, with every indication of a speedy and complete recovery. A deposition of ossific matter now gives evidence of a fracture.

To some, this case may not seem remarkable. To me it is interesting, and more especially so, because many physicians believe such an occurrence impossible without fatal consequences.

Lawrence, May 19th, $18 j 1$.

[Dr. Arcorr has a rare faculty of collecting curious medical facts, and great ingenuity in explaining them. The case alluded to below is an interesting one, and is worthy of being placed upon record. We know of no particular reason to doubt the statements in regard to it, but still it would be gratifying to learn something further from the physicians of Nantucket, who must be supposed to have better opportunities than others of knowing the particulars of so remarkable a case. But, if correct in every point, "one swallow does not make a summer;" and as one patient or even twenty may get well under homœopathic treatment without proving the efficacy of infinitesimal doses, so the solitary case of Mr. Robinson can have litile weight in disproving the old-fashioned doctrine that something besides air and water and "a strong will" are needed for the nourishment, at least, of the human body._- ED.]

\section{S'TARVING OUT DYSPEPSIA.}

\section{To the Editor of the Boston Medical and Surgical Journal.}

SIR,-In my communication under the above head, in the Journal of May 21, I studied brevity to an extent which has been regretted by some 